
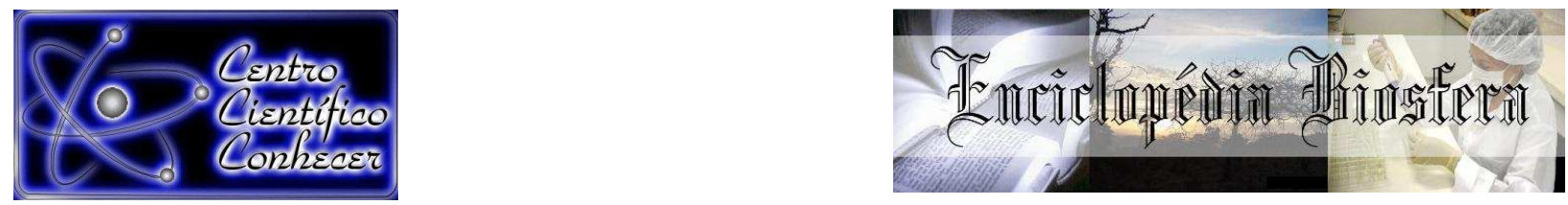

\title{
EQUAÇÕES DE BIOMASSA PARA AROEIRA-PIMENTEIRA (Schinus terebinthifolius RADDI)
}

Mateus Niroh Inoue Sanquetta ${ }^{1}$; Carlos Roberto Sanquetta ${ }^{2}$; Aline Durigan ${ }^{1}$; Ana Paula Dalla Corte $^{3}$; Vinicius Morais Coutinho ${ }^{1}$

\section{1 - Acadêmico de Engenharia Florestal da Universidade Federal do Paraná (mateus.sanquetta@hotmail.com)}

2 - Eng. Florestal, Ph.D., Professor da Universidade Federal do Paraná

3 - Eng. Florestal, Dr., Professora da Universidade Federal do Paraná Centro BIOFIX - Universidade Federal do Paraná - UFPR

Av. Lothário Meissner, 900 - Jardim Botânico

Curitiba - PR, 80.210-170 - Brasil

Recebido em: 08/09/2015 - Aprovado em: 14/11/2015 - Publicado em: 01/12/2015 DOI: http://dx.doi.org/10.18677/Enciclopedia_Biosfera_2015_248

Este estudo objetivou avaliar quatro modelos de regressão para estimar a biomassa seca aérea de Schinus terebinthifolius. Os dados foram coletados no município de Pinhais, PR, em um plantio estabelecido em 2003. Mensurou-se diretamente o dap (diâmetro à altura do peito) e a ht (altura total) de 30 árvores, que após o corte e a derrubada foram objeto de quantificação de biomassa verde pelo método direto. Foram selecionadas porções amostrais representativas da biomassa, com o objetivo de realizar as determinações da biomassa seca, estas foram levadas à em estufa a $70^{\circ} \mathrm{C}$ até peso constante para obter a biomassa seca aérea de cada indivíduo (ba). Foram testados quatro modelos matemáticos, a saber: Kopezky-Gehrhardt, Spurr, Husch e Schumacher-Hall. Para os modelos logarítmicos aplicou-se o Fator de Correção de Meyer. As equações foram obtidas pelo método dos mínimos quadrados ordinários. Foram utilizados três critérios de seleção de modelos para o julgamento do melhor entre eles: coeficiente de determinação ajustado $\left(R_{\text {aj. }}^{2}\right.$ ) e erro padrão da estimativa em porcentagem $\left(S_{y x \%}\right)$ e a análise gráfica dos resíduos. 0 conjunto de dados avaliado apresentou dap médio de $8,02 \pm 1,75 \mathrm{~cm}$ e altura total média de 7,52 $\pm 0,65$, enquanto a biomassa aérea seca variou de $16,38 \pm 7,94 \mathrm{~kg}$. A equação selecionada foi $b a=-0,325050+0,261156$. dap ${ }^{2}$, correspondente ao modelo de Kopezky-Gehrhardt. Os valores observados para $R_{a j}^{2}$. e $S_{y x \%}$ do modelo selecionado foram 0,781 e $25,54 \%$, respectivamente. Todos os modelos geraram estimativas satisfatórias, mas que o modelo de Kopezky-Gehrhardt apresentou leve superioridade em relação aos demais e que esse modelo é mais simples por requerer apenas o dap como variável independente, sendo recomendado para uso.

PALAVRAS-CHAVE: ajuste, análise de resíduos, modelagem, massa seca, regressão. 


\title{
ABOVEGROUND BIOMASS EQUATIONS FOR BRAZILIAN PEPERTREE (SCHINUS TEREBINTHIFOLIUS RADDI)
}

\begin{abstract}
This study aimed to evaluate four regression models to estimate aboveground dry biomass of Schinus terebinthifolius. Data were collected in the municipality of Pinhais, PR, in a plantation established in 2003. It is measured directly $d b h$ (diameter at breast height) and $h t$ (overall height) of thirty trees, which after cutting were subject to quantification of aboveground fresh biomass by direct method. Representative sampling portions in biomass were selected, weighed and oven dried at $70^{\circ} \mathrm{C}$ until constant weight to obtain the aboveground dry biomass of each individual (ba). Four mathematical models were tested, namely: Kopezky-Gehrhardt, Spurr, Husch and Schumacher-Hall. For the logarithmic models we applied the Meyer Correction Factor. The equations were obtained by the ordinary least squares method. Three criteria for selection of models were employed to judge the best among them: adjusted coefficient of determination $\left(R_{\text {adj. }}^{2}\right)$ and standard error of estimate in percentage $\left(S_{y x}\right)$ and graphical residual analysis. The data evaluated had an average $d b h$ of $8.02 \pm 1.75 \mathrm{~cm}$ and total mean height of $7.52 \pm 0.65$, where as dry biomass ranged from $16.38 \pm 7.94 \mathrm{~kg}$. The selected equation was ba $=-0.325050+$ 0.261156 . $d b h^{2}$, corresponding to the Kopezky-Gehrhardt model. The observed values of $R_{\text {adj. }}^{2}$ and $S_{y x}$ of the selected model were 0.781 and $25.54 \%$, respectively. All models yielded satisfactory estimates, but the Kopezky-Gehrhardt model showed a slight superiority over the others and that this model is simpler because it only requires $d b h$ as an independent variable, being recommended to use.
\end{abstract}

KEYWORDS: fit, residual analysis, modeling, dry weight, regression

\section{INTRODUÇÃO}

As florestas representam um dos recursos naturais mais importantes para a humanidade. Além de produtos, geram serviços ambientais vitais como por exemplo, os ciclos de ar e água, clima, polinização, e ciclagem de nutrientes (HENRY et al., 2014), entre os quais a capacidade de armazenar carbono por meio da fixação biológica via fotossíntese.

Árvores acumulam em suas estruturas, além do carbono, outros elementos determinantes nos ciclos naturais e também para a qualidade de vida das pessoas. Por isso, o interesse pelos estudos sobre a biomassa e conteúdo de carbono em florestas é crescente (SILVEIRA, 2008). O interesse cresce também na medida em que os produtos provenientes de biomassa florestal podem ser utilizados de forma sustentável em substituição aos combustíveis fósseis e, de forma análoga, também estocando carbono.

A biomassa é uma variável fundamental na quantificação do estoque e da dinâmica de carbono em florestas. A maior parte do carbono de uma floresta e das árvores que a compõem está concentrada nesse reservatório biológico (SANQUETTA et al., 2015).

O termo biomassa expressa a quantidade de massa de um material vegetal. Mais especificamente no âmbito florestal, o termo biomassa aplica-se a toda biomassa existente em uma floresta ou em apenas uma fração da mesma. Além deste, outros termos como fitomassa florestal e fitomassa arbórea também são utilizados (SANQUETTA, 2002). A biomassa florestal pode ser expressa por massa verde ou massa seca, sendo que a massa verde se refere ao material fresco 
amostrado, contendo uma variável proporção de água. Já a massa seca se refere a biomassa obtida após secagem em estufa (CALDEIRA, 2003).

As estimativas de biomassa e carbono em florestas podem ser feitas por métodos diretos e indiretos. Os métodos diretos consistem em pesar toda a biomassa, geralmente por processo destrutivo. Esta abordagem é inviável para aplicação em grandes áreas, devido ao tempo e ao custo de execução. Por outro lado, os métodos indiretos implicam em emprego de algum tipo de modelagem (SANQUETTA et al., 2014). Usualmente, utilizam-se equações de biomassa por meio de regressão linear, empregando variáveis como diâmetro à altura do peito, altura total ou comercial como variáveis dependentes (SOMOGYI et al., 2006). Tais equações facilitam em muito a quantificação de carbono em florestas.

Muitas espécies nativas da floresta brasileira, particularmente da Mata Atlântica, ainda não foram estudadas quanto ao potencial de estocagem de carbono. Assim, o presente estudo objetivou testar modelos matemáticos a partir de dados de biomassa aérea individual de aroeira-pimenteira (Schinus terebinthifolius), com vistas a possibilitar a estimativa indireta da biomassa dessa espécie nativa que apresenta importância comercial por possuir propriedades medicinais (GUERRA et al., 2000), além de se destacar ecologicamente em programas de recuperação de áreas degradadas (GANDARA, 2000).

\section{Área de Estudo}

\section{MATERIAL E MÉTODOS}

O presente estudo foi realizado com dados de indivíduos arbóreos provenientes do Centro de Estações Experimentais do Canguiri (CEEC), pertencente à Universidade Federal do Paraná e situado no município de Pinhas, PR. O CEEC está inserido no primeiro planalto paranaense, mais precisamente na região metropolitana de Curitiba. A área em questão possui coordenadas geográficas de 2523'30" S para latitude e 4907'30" W para longit ude, a altitude média é de $900 \mathrm{~m}$. O clima predominante segundo a classificação de Köppen é o subtropical úmido mesotérmico ( $\mathrm{Cfb})$, com verões frescos e invernos rigorosos, a temperatura média anual é de $17,5^{\circ} \mathrm{C}$, sendo a máxima de $22,5^{\circ} \mathrm{C}$ e a mín ima de $12,5^{\circ} \mathrm{C}$. A precipitação é bem distribuída em todas as estações do ano (RIBEIRO et al., 2008).

\section{Descrição da espécie}

A espécie Schinus terebinthifolius Raddi, mais conhecida como aroeirapimenteira, aroeira vermelha ou apenas como aroeira, é nativa do Brasil e pertence à família Anacardiaceae, tendo ocorrência natural no sul do continente americano, marcadamente na costa brasileira, sendo bastante comum na Mata Atlântica (MARTORELLI et al., 2011).

Conhecida por suas propriedades medicinais e alimentícias, é recomendada também para a recuperação de áreas degradadas (FERRETI et al. 1995). No entanto, esta espécie também vem sendo categorizada como invasora (D'AVILA et al., 2010) em certas circunstâncias, podendo trazer desequilíbrio ambiental devido à ampla dispersão e rápido crescimento.

\section{Coleta de Dados em Campo}

Durante o mês de dezembro do ano de 2014 foi realizada uma atividade em campo em que 30 indivíduos da espécie Schinus terebinthifolius foram selecionados e abatidos. Os indivíduos pertenciam a um plantio experimental estabelecido em 2003. As árvores tiveram as variáveis diâmetro à altura do peito ou 1,30 $\mathrm{m}$ acima do 
nível do solo (dap) e altura total $(h t)$ tomadas a partir de medições diretas com auxílio de fita métrica e trena. Em seguida, as árvores foram segmentadas em fuste, galhos grossos, galhos finos e folhagem, e pesadas individualmente, obtendo-se assim as respectivas biomassas aéreas individuais frescas.

Amostras de cerca de $200 \mathrm{~g}$ foram retiradas das frações da biomassa e pesadas em estufa a $70^{\circ} \mathrm{C}$ até peso constante. As relações entre as biomassas secas e frescas das amostras foram então utilizadas para calcular a biomassa aérea seca total de cada árvore $(b a)$.

\section{Modelagem da Biomassa}

A estimativa da biomassa seca aérea foi realizada pelo método da regressão linear simples e múltipla, empregando-se o método de mínimos quadrados ordinários. Para tal, foram utilizados quatro diferentes modelos usuais no meio florestal que utilizam variáveis independentes de dap e ht (Quadro 1). Entre os modelos testados, três são de simples entrada (utiliza apenas uma variável independente) e um de dupla entrada (utiliza duas variáveis independentes).

QUADRO 1 - Modelos e equações para a estimativa da biomassa aérea seca de Schinus terebinthifolius

\begin{tabular}{ccc}
\hline & Modelos & Equações \\
\hline$(1)$ & Kopezky-Gehrhardt & $b a=\beta_{0}+\beta_{1} \cdot$ dap ${ }^{2}+e_{i}$ \\
$(2)$ & Spurr & $b a=\beta_{0}+\beta_{1} \cdot\left(\right.$ dap $\left.{ }^{2} \cdot h t\right)+e_{i}$ \\
$(3)$ & Husch & $\ln (b a)=\beta_{0}+\beta_{1} \cdot \ln ($ dap $)+e_{i}$ \\
$(4)$ & Schumacher-Hall & $\ln ($ ba $)=\beta_{0}+\beta_{1} \cdot \ln ($ dap $)+\beta_{2} \cdot \ln (h t)+e_{i}$ \\
\hline
\end{tabular}

Em que: $b a=$ biomassa aérea individual $(\mathrm{kg}) ; \mathrm{ln}=$ logaritmo neperiano; $\beta_{0} \ldots \beta_{n}=$ coeficientes obtidos através do método de regressão linear; dap = diâmetro a $1,30 \mathrm{~m}$ do solo $(\mathrm{cm}) ; h t=$ altura total $(\mathrm{m}) ; e_{i}$ $=$ erro associado

Os modelos logarítmicos (3 e 4) estimam a variável dependente (ba) em unidades logarítmicas e, por este motivo, a comparação com os modelos aritméticos só pode ser realizada com conversões. Para esse fim foi realizada a aplicação do Fator de Correção de Meyer, de acordo com a Equação 5.

$$
F C M=e^{\left(0,5^{\star} S y x\right)}
$$

Em que: $S y x=$ erro padrão da estimativa absoluto $(\mathrm{kg})$.

Os modelos foram avaliados segundo três critérios de seleção (quadro 2), sendo estes o coeficiente de determinação ajustado $\left(R_{\text {aj. }}^{2}\right)$, o erro padrão da estimativa $\left(S_{y x}\right)$ e a análise gráfica dos resíduos. O coeficiente de determinação ajustado expressa, em valores entre zero e um ou em porcentagem, a capacidade explicativa do modelo levando-se em consideração os dados observados.

O erro padrão da estimativa, por sua vez, indica a dispersão média entre os valores estimados e os observados. Para as equações que a variável dependente sofreu linearização (3 e 4), foi necessário recalcular o erro padrão residual, deixando todos os valores nas mesmas dimensões, possibilitando assim a comparação com os demais modelos. 
Por fim, empregou-se a análise gráfica dos resíduos, indicador fundamental em julgamento de modelos, pois permite verificar possíveis tendências na distribuição dos resíduos. Da mesma forma, apresenta as dispersões percentuais dos valores estimados em relação aos observados, mas de forma individual. Uma análise gráfica perfeita teria todos os seus pontos localizados no eixo das abcissas.

QUADRO 2 - Critérios de seleção de modelos utilizados para modelagem da biomassa aérea seca total de indivíduos de Schinus terebinthifolius Critério Formulação

$$
R_{a j .}^{2}=1-\frac{(n-1)}{(n-k)}\left(1-R^{2}\right)
$$

Coeficiente de determinação ajustado

$$
R^{2}=1-\frac{\sum_{i=1}^{n}\left(b a_{i}-\hat{b} a_{i}\right)^{2}}{\sum_{i=1}^{n}\left(b a_{i}-\bar{b} a\right)^{2}}
$$

$$
S_{y x \%}=\frac{S_{y x}}{\bar{b} a} * 100
$$

Erro padrão da estimativa em

$$
\begin{gathered}
S_{y x}=\sqrt{\frac{\sum_{i=1}^{n}\left(b a_{i}-\hat{b} a_{i}\right)^{2}}{n-p}} \\
e_{i}=\frac{\left(b a_{i}-\hat{b} a_{i}\right)}{b a_{i}} * 100
\end{gathered}
$$

Em que:

$n=$ número de observações;

$p=$ número de coeficientes do modelo;

$b a_{i}=$ biomassa aérea individual $(\mathrm{kg})$;

$\hat{b} a_{i}=$ biomassa aérea estimada $(\mathrm{kg})$;

$\bar{b} a=$ biomassa aérea média $(\mathrm{kg})$.

\section{Dados Dendrométricos das Plantas}

\section{RESULTADOS E DISCUSSÃO}

Os indivíduos estudados possuem dimensões médias para o dap de $8,02 \pm 1,75 \mathrm{~cm}$ e de altura total de $7,52 \pm 0,65 \mathrm{~m}$. No tocante a biomassa aérea

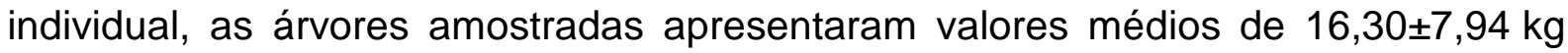
(Tabela 1).

Não existem estudos na literatura que versam sobre as dimensões atingidas por plantas de aroeira-pimenteira com idade conhecida em plantios uniformes. Normalmente a espécie é encontrada em floresta nativa ou em plantios mistos de restauração ecológica. A espécie, apesar de possuir potencial econômico, não é plantada em escala comercial no Brasil.

Da mesma forma, inexistem estudos sobre a produção de biomassa para indivíduos de aroeira-pimenteira, principalmente em plantações homogêneas e 
equiâneas. Relatos foram observados em estudos realizados em fragmentos de florestas naturais nos quais a espécie ocorre, a exemplo do estudo realizado por CALDEIRA (2003), em que o autor realizou a determinação da biomassa de diferentes espécies em um fragmento de Floresta Ombrófila Mista Montana, no município de General Carneiro, PR. Entre as diferentes espécies estudadas pelo autor, a $S$. terebinthifolius ocorreu com biomassa individual média de 9,28 kg, valor este inferior aos valores médios observados para os indivíduos estudados.

TABELA 1 - Variáveis dendrométricas de árvores de Schinus terebinthifolius

\begin{tabular}{ccc}
\hline Estatística & Variável & Valores \\
\hline Média \pm Desvio Padrão & dap $(\mathrm{cm})$ & $8,02 \pm 1,75$ \\
& $h t(\mathrm{~m})$ & $7,52 \pm 0,65$ \\
$\mathrm{~N}^{0}$ de indivíduos & ba $(\mathrm{kg})$ & $16,30 \pm 7,94$ \\
\hline
\end{tabular}

\section{Estimativa de Biomassa}

Para a estimativa da biomassa da aroeira-pimenteira foram testados diferentes modelos. A seleção do modelo com os melhores parâmetros foi realizada com base nos critérios de seleção previamente descritos.

Observou-se, que todos os modelos apresentaram padrão semelhante de acurácia (Tabela 2). De maneira geral, todos os modelos apresentaram erros padrão da estimativa semelhantes. Os valores de $R_{a j}^{2}$ apontam que o Modelo (3), de Kopezky-Gehrhardt, como o mais acurado. Para o $S_{y x} \%$, a melhor performance também foi obtida com o mesmo modelo.

No que concerne à análise gráfica de resíduos, se observou que não houve tendência nas estimativas, ou seja, viés de subestimativa ou superestimativa ao aplicar as equações obtidas via regressão. Graficamente a distribuição de resíduos do Modelo (3) foi a mais equilibrada, com resíduos localizados de forma balanceada na parte superior e inferior do gráfico e confinados entre -40 e $+40 \%$ (Figura 1).

TABELA 2 - Ajustes das equações para a estimativa de biomassa seca individual acima do solo em árvores de Schinus terebinthifolius

\begin{tabular}{cccccc}
\hline Equação & $\beta_{0}$ & $\beta_{1}$ & $\beta_{2}$ & $S_{y x} \%$ & $R_{a j .}^{2}$ \\
\hline$(1)$ & $-0,325050$ & 0,261156 & - & 25,54 & 0,781 \\
$(2)$ & 1,766874 & 0,030107 & - & 25,95 & 0,759 \\
$(3)$ & $-1,338270$ & 1,976655 & - & 26,38 & 0,750 \\
$(4)$ & $-2,364490$ & 1,862581 & 0,626321 & 27,59 & 0,761
\end{tabular}

Os ajustes obtidos foram satisfatórios, de forma geral, principalmente tratando-se de uma espécie com estudos praticamente inexistentes na literatura nacional. RATUCHNE (2015) desenvolveu um trabalho de ajuste de equações de biomassa para um fragmento de Floresta Ombrófila mista no Estado do Paraná. Nesse estudo, o autor obteve valores de $R_{\text {aj. }}^{2}$ variando de 0,977 a 0,981 , superiores aos determinados. 
No entanto, os valores para $S_{y x}$ foram superiores quando comparados aos obtidos no presente estudo. De forma geral, os valores que o RATUCHNE (2015) determinou variaram de $47,83 \%$ a $53,67 \%$, consideravelmente superiores aos deste estudo. Esta diferença pode ser explicada pelo fato de mais espécies terem sido analisadas em seu estudo.

a)

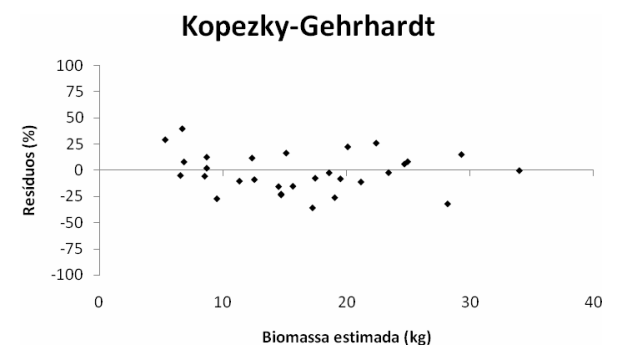

c)

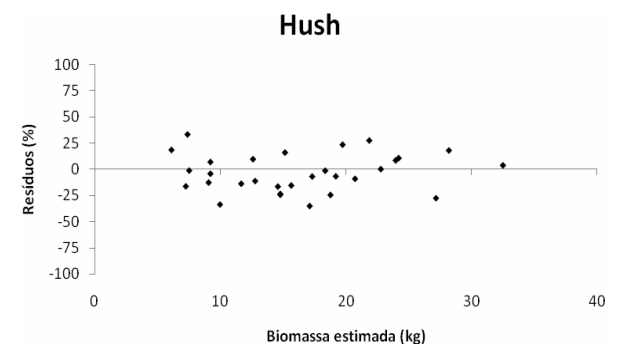

b)

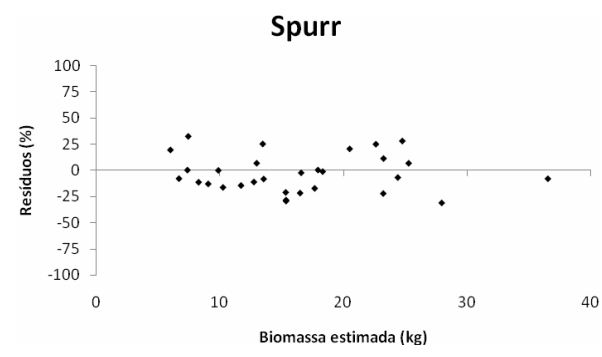

d)

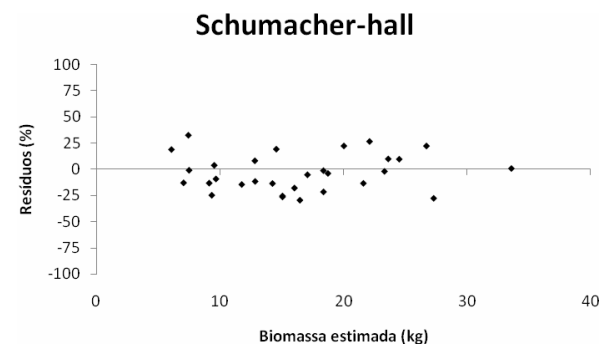

FIGURA 1 - Distribuição gráfica dos resíduos em ajuste de biomassa seca acima do solo em árvores de Schinus terebinthifolius

BARBEIRO et al. (2009) testaram modelos alométricos para biomassa seca de diferentes compartimentos em Nectandra grandiflora, a partir de variáveis de fácil mensuração. Os autores concluíram que as biomassas secas, total e do fuste podem ser estimadas satisfatoriamente, o mesmo não ocorreeu com a folhagem. $O$ modelo mais adequado leva como variável independente o dap e a variável combinada dap $h t$, ambos na forma logarítmica. Os valores de $R^{2} a j$. e $S_{y x \%}$ foram 0,9844 e $13,71 \%$, respectivamente.

MELO et al. (2014) avaliaram modelos para caixeta (Tabebuia cassinoides (Lam.) DC.) no Paraná e encontraram que modelos simples podem ser utilizados para expressar a biomassa e o carbono individual. No caso, o modelo linear foi o mais adequado, com $R^{2}$ aj. de 0,96 e $S_{y x \%}$ de 7,94 .

SANQUETTA et al. (2014) analisaram quatro modelos para Araucaria angustifolia (Bert.) O. Ktze., considerando a modelagem direta do estoque individual de carbono e a modelagem do volume e a aplicação de fatores de expansão. Entre os modelos avaliados o de Husch (de simples entrada) se mostrou o mais acurado. Resultados semelhantes foram obtidos por RATUCHNE et al. (2015).

\section{CONCLUSÕES}

- Os quatro modelos testados tiveram desempenhos satisfatórios, considerando se tratar de uma espécie nativa sem melhoramento genético e com grandes 
variações arquitetônicas, que refletem em variabilidade na estocagem de biomassa;

- O melhor modelo entre os quatro testados foi o de Kopezky-Gehrhardt, que é simples e utiliza como variável independente apenas o diâmetro à altura do peito, sendo, portanto, de fácil aplicação;

- A análise gráfica de resíduos apontou que os modelos testados não apresentaram tendências, particularmente o modelo selecionado mostrou equilíbrio na distribuição dos resíduos;

- Schinus terebinthifolius, apesar de ter grande importância por suas características medicinais e alimentícias, carece de estudos direcionados ao seu potencial para produção em escala comercial e prestação de serviços ecossistêmicos.

\section{REFERÊNCIAS}

BARBEIRO, L. S. S.; VIEIRA, G.; SANQUETTA, C. R. Equações para estimativa da biomassa individual de Nectandra grandiflora Ness (canela-amarela). Floresta, v. 39, n. 4, p. 833-843, 2009.

CALDEIRA, M. V. W. Determinação de biomassa e nutrientes em uma Floresta Ombrófila Mista Montana em General Carneiro, Paraná. 176f. Tese (Doutorado em Ciências Florestais) - Universidade Federal do Paraná, Curitiba, PR, 2003.

D'AVILA, G.; GOMES JR., A.; CANARY, A. C.; BUGONI, L. The role of avian frugivores on germination and potential seed dispersal of the Brazilian Pepper Schinus terebinthifolius. Biota Neotropica, v. 10, n. 3, p.45-51, 2010.

FERRETTI, A. R.; KAGEYAMA, P. Y.; ÁRBOEZ, G. F.; SANTOS, J. D.; BARROS, M. I. A.; LORZA, R. F.; OLIVEIRA, C. Classificação das espécies arbóreas em grupos ecofisiológicos para revegetação com nativas no estado de São Paulo. Florestar Estatístico, v. 3, n. 7, p. 73-77, 1995.

HENRY, M. H.; JARA, M. C.; RÉJOU-MÉCHAIN, M.; PIOTTO, D.; FUENTES, J. M. M.; WAYSON, C.; GUIER, F. A.; LOMBIS, H. C.; LÓPEZ, E. D.; LARA, R. C.; ROKAS, K. C.; PASQUEL, J. D. A.; MONTOYA, A. D.; VEGA, J. F.; GALO, A. J.; LÓPEZA, O. R.; MARKLUND, L.G.; MILLA, F.; CAHIDEZ, J.J.N.; MALAVASSI, E.O.; PEREZ, J.; ZEA, C.R.; GARCÍA, L.R.; PONS, R.R.; SANQUETTA, C.; SCOTT, C.; WESTFALL, J.; ZAPATA-CUARTAS, M.; SAINT-ANDRÉ, L. Recommendations for the use of tree models to estimate national forest biomass and assess their uncertainty. Annals of Forest Science, v. 72, n. 6, p.1-9, 2014.

MARTORELLI, S. B. F.; PINHEIRO, A.L.B.; HIGINO, J.S.; BRAVO, F. Efeito antiinflamatório e cicatrizante de extrato de hidroalcoólico de Schinus terebinthifolius Raddi (aroeira) a $30 \%$ em ora base - estudo "in vivo". International Journal of Dentistry, v. 10, n. 2, p.80-90, 2011.

MELO, L. C.; SANQUETTA, C. R.; DALlA CORTE, A. P.; HENTZ, A. K. Estimativa de biomassa e carbono total para árvores de caixeta no Paraná. Pesquisa Florestal Brasileira, v. 34, n. 77, p. 21-29, 2014. 
RATUCHNE, L. C. Biomassa e carbono: equações e dinâmica em fragmentos de Floresta Ombrófila Mista no Paraná. 102f. Tese (Doutorado em Engenharia Florestal) - Universidade Federal do Paraná, Curitiba, PR, 2015.

RATUCHNE, L. C.; BRISTOLIM, J. C.; KOEHLER, H. S.; WATZLAWICK, L. F.; SANQUETTA, C. R.; SCHAMNE, P. A. Quantificação de carbono florestal em povoamentos de Araucaria angustifolia no sudoeste do estado do Paraná. Ambiência, v. 11, n. 2 p. 321-335, 2015.

RIBEIRO, L.; KOPROSKI, L. P.; STOLLE, L.; LINGNAU, C.; SOARES, R. V.; BATISTA, A. C. Zoneamento de riscos de incêndios florestais para a Fazenda Experimental do Canguiri, Pinhais (PR). Floresta, v.38, n.3, p. 561- 572, 2008.

SANQUETTA, C. R. Métodos de determinação de biomassa florestal. In: SANQUETTA, C. R.; WATZLAWICK, L. F.; BALBINOT, R. M.; ZILIOTTO, M. A. B.; GOMES, F. S. (Ed.). As florestas e o carbono. Curitiba: UFPR, p. 119-140.2002.

SANQUETTA, C. R.; DALLA CORTE, A. P.; MOGNON, F.; MAAS, G. C. B.; RODRIGUES, A. L. Estimativa de carbono individual para Araucaria angustifolia. Pesquisa Agropecuária Brasileira, v. 44, n. 1, p. 1-8, 2014.

SANQUETTA, C. R.; WOJCIECHOWSKI, J.; DALLA CORTE, A. P.; BEHLING, A.; PÉLLICO NETTO, S. P; RODRIGUES, A. L.; SANQUETTA, M. N. I. Comparison of data mining and allometric model in estimation of tree biomass. BMC Bioinformatics, v. 16, n.247, p. 1-9, 2015.

SILVEIRA, P.; KOEHLER, H. S.; SANQUETTA, C. R.; ARCE, J. E. Estado da arte na estimativa de biomassa e carbono em formações florestais. Floresta, v. 38, n. 1, p. 185-206, 2008.

SOMOGYI, Z.; CIENCIALA, E.; MÄKIPÄÄ, R.; MUUKKONEN, P.; LEHTONEN A.; WEISS, P. Indirect methods of large forest biomass estimation. European Journal of Forest Research, v. 126, n. 2, p.197-207, 2006. 